CORRECTION

https://doi.org/10.1038/s41586-019-0930-z

\title{
Author Correction: Systems of mechanized and reactive droplets powered by multi-responsive
} surfactants

Zhijie Yang, Jingjing Wei, Yaroslav I. Sobolev \& Bartosz A. Grzybowski

Correction to: Nature https://doi.org/10.1038/nature25137, published online 10 January 2018.

In the top right panel of Fig. 1a of this Letter, we inadvertently showed the chemical structure of elaidic acid (trans-9-octadecenoic acid), rather than that of oleic acid (cis-9-octadecenoic acid) used in the paper. Figure 1 of this Amendment shows the original and corrected structures. This error has not been corrected online.

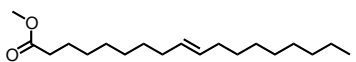

b

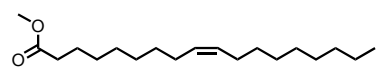

Fig. 1 | This figure shows the original and corrected top structure of the right panel of Fig. 1a of the original Letter. a, The as-published structure of trans-9-octadecenoic acid. $\mathbf{b}$, The structure of cis-9-octadecenoic acid that should have been published. 